\title{
Antibiotic stewardship, Calgary 1995-1997: Regionalization, restructuring and retrenchment
}

\author{
TJ Louie MD FRCPC, RR Read MD PhD FRCPC
}

$\mathrm{H}$ ospitals in the Calgary area have undergone a decade of change as a result of provincial and local initiatives to provide the same or better health care with fewer dollars. The new system includes 1850 beds at four hospitals, a continuing care sector coordinating nursing home and ambulatory care services, and population health and public health services. The reorganization is patient-ocused, using a "hospital without walls" concept to promote linkages and efficiencies in health care delivery.

Before the elimination of individual hospital boards and the creation of one regional health board in 1994, each of the hospital groups had organized antibiotic utilization programs with the primary aims of making better use of antibiotics and taking efforts towards cost containment. Programs were more intensive and comprehensive at the teaching institutions. Community hospitals that adopted policies according to local needs. Being the primary teaching hospital in Calgary region, the Foothills Hospital practised a series of antimicrobial use and restriction guidelines via the Online Survey and Certification Reporting (OSCAR) hospital computer system. At the Calgary General and Alberta Children's hospitals, the infectious diseases consultation service was used to restrict the excessive use of broad-spectrum and expensive antimicrobial agents. Four of the five hospitals had antibiotic utilization subcommittees that reported to pharmacy and therapeutics committees. Three part-time drug use evaluation (DUE) pharmacists ( 0.25 to 0.6 full-time equivalent) were available to monitor antimicrobial use and fulfill an educational function. Specific antibiotic stewardship activities included writing pharmacy newsletters, monitoring intravenous to oral stepdown, tracking antimicrobial costs, instituting therapeutic interchanges, standardizing antibiotic regimens, and reviewing cephalosporin, vancomycin, clindamycin and imipenem/cilastatin use. Individual cost cards indicating antimicrobial susceptibilities were updated annually by each hospital group.

Plans for downsizing and reorganizing hospital services were made between 1991 and 1994. Three of seven hospitals were closed between 1995 and 1997. A \$100 million renovation and building program was implemented and completed to upgrade remaining facilities.

The hospital and community laboratory systems have been merged into a public/private service company, and all microbiology services have been consolidated into one central 'core' laboratory. While disadvantages may be associated with not having a microbiology laboratory in each of the hospitals, the centralized laboratory can provide important advantages withregard to tracking and monitoring antibiotic resistance. Further examples of the restructuring of hospital services include the consolidation of all pharmacies under one regional administration, and the reorganization of the individual antibiotic utilization subcommittees into one regional, antibiotic utilization, working group. Although the majority of antibiotic use policies have been retained during hospital consolidation, standardization is now taking place. The DUE pharmacist po- 
sitions were retained at three of the four remaining hospitals. While no new resources have become available to address antibiotic stewardship initiatives, it is fortunate that both the DUE and infection control resources have survived the restructuring process without major budgetary reductions. Both of these parts of the health care system can be redirected at antibiotic stewardship functions in partnership with the laboratories, public health and the Faculty of Medicine at the University of Calgary.

Antibiotic-resistant organisms are becoming more prevalent locally; however, there are differences from trends in central Canada. Methicillin-resistant Staphylococcus aureus is sporadic and so far there have been no outbreaks since the 1991 outbreak at the Calgary General Hospital. A small outbreak of vancomycin-resistant enterococci occurred at the Foothills Hospital in mid-1997. Vigorous cohorting, isolation and screening contained the outbreak to five colonized patients. Avoidance of unnecessary cephalosporin and vancomycin use, and a recommendation by the Infectious Diseases Service to use more beta-lactam/beta-lactamase combinations following the experience of Quale et al (1) were proposed as antibiotic strategies to reduce ecological selective pressure. Penicillin resistance in Streptococcus pneumoniae is at the $20 \%$ level at present. The paediatric infectious disease division has undertaken an Alberta Heritage Foundation-supported study on the epidemiology of $S$ pneumoniae. Multiply resistant, Gram-negative bacterial infections including infections by extended spectrum beta-lactamase producing organisms have recently emerged in an intensive care setting. A regionwide surveillance program is being discussed for this group of pathogens.

Future directions of antibiotic stewardship in the Calgary region include the following:

- re-establishing a list of priorities/activities/program directions;

- seeking partnerships with existing organizations public, private and volunteer - to combat antibiotic resistance;
- establishing links with other regional, provincial and national organizations;

- ensuring that stewardship activities are appropriately funded (there is no specific medical funding for stewardship activities, although all members of the infectious diseases physician and microbiologist groups have volunteered time and interest);

- standardizing and rationalizing antimicrobial use policies throughout the region;

- establishing guidelines/protocols for treatment of common infections;

- initiating control programs/projects at 'pressure points' where antibiotic resistance is likely to be generated,ie, intensive care units, immunocompromised host units, day care centres, and front line primary care clinics;

- aiming DUE activities at specific antibiotics such that 'disease-specific' drugs are evaluated on an ongoing basis.

\section{CONCLUSIONS}

Restructuring, 'down-sizing' or 'right-sizing', reorganizing or regionalizing health systems can slow down, distract or misplace the organizational focus on antibiotic stewardship. The responsible parties at all levels need to heed the urgency to pull together available resources, to add new resources if necessary and to prioritize activities in the present crisis of increasing antibiotic resistance.

ACKNOWLEDGEMENTS: The continued Department of Pharmacy support of S Long, V Radkovich and L Heule is appreciated.

\section{REFERENCES}

1. Quale J, Landman D, Saurina G, Atwood E, DiTore V, Patel K. Manipulation of a hospital antimicrobial formulary to control an outbreak of vancomycin-resistant enterococci. Clin Infect Dis 1996;23:1020-5. 


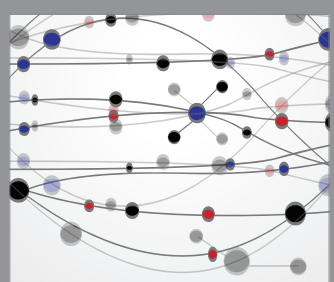

The Scientific World Journal
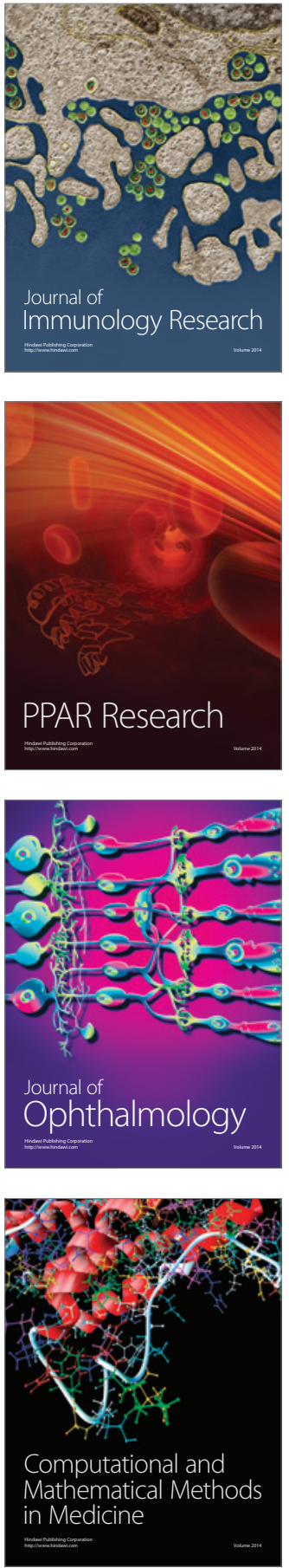

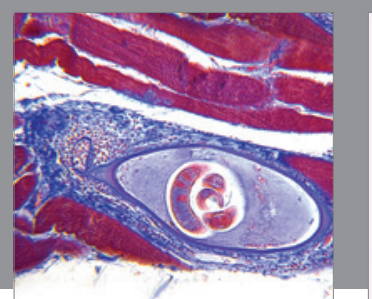

Gastroenterology Research and Practice

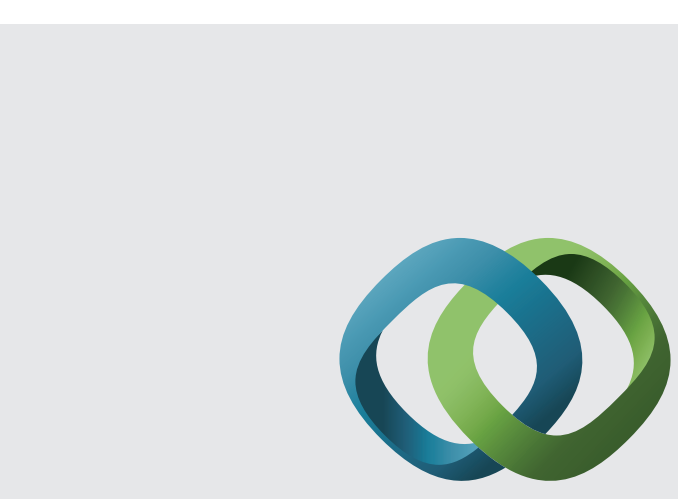

\section{Hindawi}

Submit your manuscripts at

http://www.hindawi.com
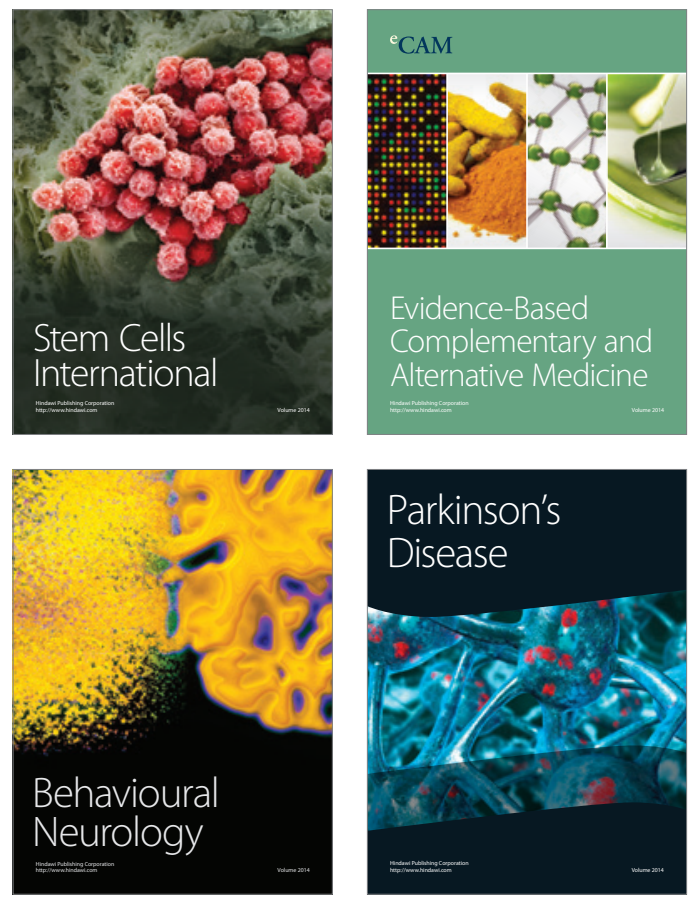
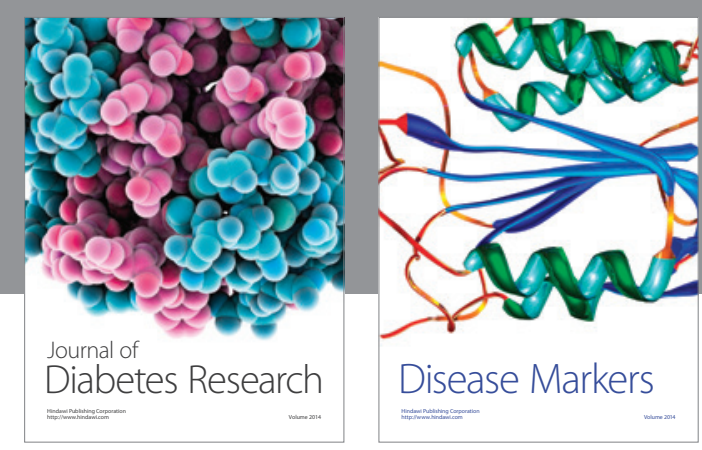

Disease Markers
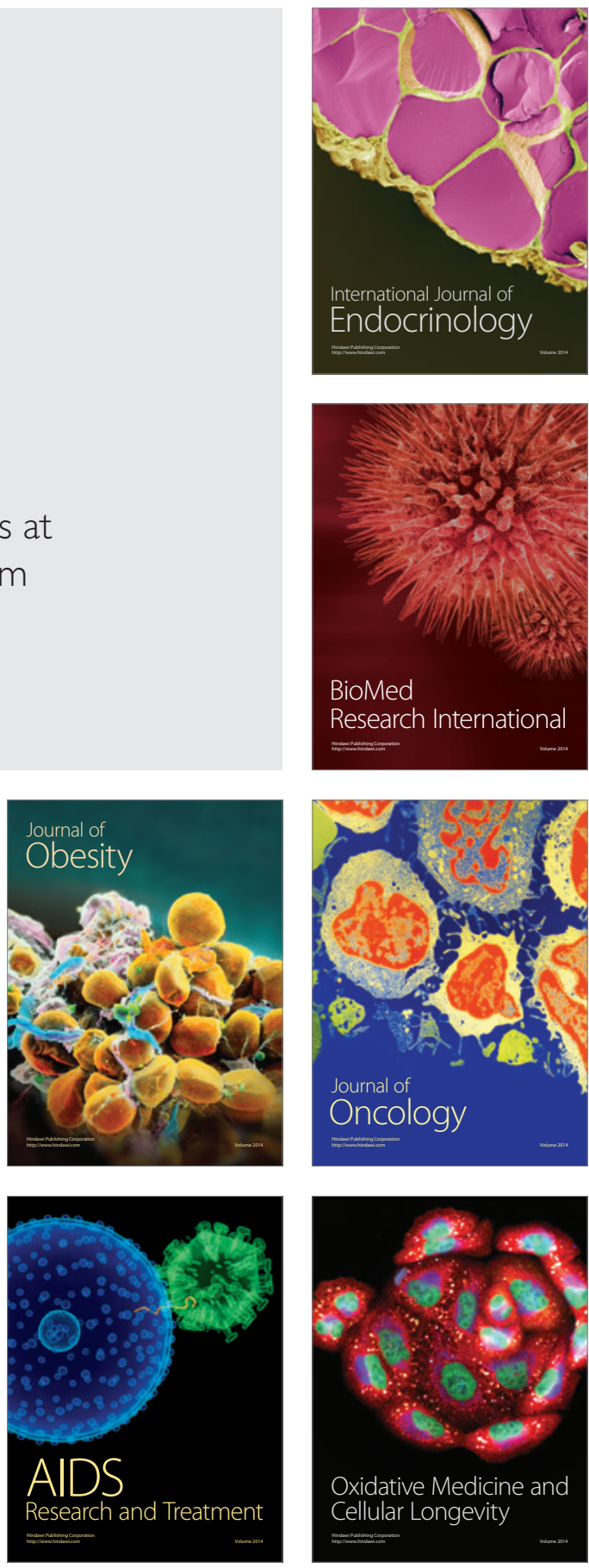